\title{
Evaluation of Myopathy Risk for HMG-CoA Reductase Inhibitors by Urethane Infusion Method
}

\author{
Kenji Matsuyama,* Kimiko NaKagawa, Aki Nakai, Yuriko Konishi, Mayumi Nishikata, \\ Hiromi TANAKA, and Takahiro UCHIDA \\ School of Pharmaceutical Sciences, Mukogawa Women's University, 11-68 Koshien 9-Bancho, Nishinomiya 663-8179, \\ Japan. Received July 18, 2001; accepted December 6, 2001
}

\begin{abstract}
Purpose of the present study was to evaluate the myopathy risk using a urethane infusion method following oral administration of five kinds of commercial HMG-CoA (3-hydroxy-3-methylglutaryl-coenzyme A) reductase inhibitors (HCRIs), (pravastatin (PV), simvastatin (SV), cerivastatin (CeV), atorvastatin (AV), and fluvastatin (FV)) alone or with coadministration of bezafibrate (BF). The solubility of HCRIs in various solvents was determined as a criterion of the physicochemical property. The plasma creatine phosphokinase (CPK) level as a marker of myopathy in normal rats was screened under urethane infusion after oral administration of HCRI alone or with BF coadministration. Also, renal tissue specimens were prepared and the myoglobin remaining in the tissue was visualized by the labeled avidin-biotin technique. The plasma CPK level in normal rats under urethane infusion following oral administration of five kinds of HCRI increased as the dose of HCRI increased, and coadministration of BF further increased the CPK level for each drug. The risk of myopathy evaluated from the CPK level was ranked as follows: $\mathrm{CeV}>\mathrm{FV}>\mathrm{AV}>\mathrm{SV}>\mathrm{PV}$. Myoglobin deposition was observed in the cast of proximal tubules, cytoplasm of distal tubules and collecting ducts of rat kidney extracted from rats treated with HCRIs under urethane infusion. Histopathological findings showed that the extent of myoglobin deposition increased on coadministration of $B F$ with each drug. The correlation was found for myopathy risk evaluated by CPK level using the urethane infusion method and drug lipophilicity, i.e., the water/n-octanol partition coefficient except for the case of SV. Histopathological findings for the kidney following HCRI treatment also reflected the CPK level in rats under urethane infusion.
\end{abstract}

Key words myopathy; pravastatin; simvastatin; cerivastatin; atorvastatin; fluvastatin

Various hypolipodemic agents are widely used in clinical settings to decrease low-density cholesterol (LDL) or triglyceride concentration in human plasma. Recently, several HMG-CoA (3-hydroxy-3-methylglutaryl coenzyme A) reductase inhibitors (HCRI) have been marketed., ${ }^{1,2}$ The disadvantage of these drugs is the risk of myopathy in patients taking them. Unfortunately, there has been no quantitative method for evaluating their myopathy risk.

We previously proposed a quick and convenient screening system for evaluating the risk of myopathy following a single oral administration of pravastatin (PV) as a representative HCRI alone or coadministration with bezafibrate (BF) by screening the creatine phosphokinase (CPK) level in rats. $\left.{ }^{3}\right)$ This screening method was based on the finding that rat plasma CPK level dramatically increases after oral administration of BF under urethane infusion. Anesthetic agents such as halothane generally induced various muscle failure like neuroleptic malignant syndrome. ${ }^{4)}$ In the case of neuroleptic malignant, the rhabdomyolysis was almost happened. Our pilot study also demonstrated that CPK level dramatically enhanced by urethane infusion model in the case of caffeine, ${ }^{5}$ ) which drug was known to increase $\mathrm{Ca}^{2+}$ level in the cell. $\left.{ }^{6}\right)$ Thus there are those backgrounds for urethane infusion method used in this study.

In the present study, using this urethane infusion method, we tried to evaluate the risk of myopathy by screening rat CPK level following oral administration of five kinds of HCRI alone or with BF. The HCRIs were PV, ${ }^{7)}$ simvastatin $(\mathrm{SV}),{ }^{8,9)}$ cerivastatin $(\mathrm{CeV}),{ }^{10)}$ atorvastatin $(\mathrm{AV}),{ }^{11,12)}$ and fluvastatin $(\mathrm{FV}){ }^{13)}$ We also conducted histopathological examination of the kidney extracted from rats treated with HCRIs.
The risk of myopathy was ranked, and the myopathy mechanism is discussed based on the physicochemical properties of HCRI and in vivo data.

\section{MATERIALS AND METHODS}

Materials The HCRIs PV, SV, CeV, AV, and FV and BF were kindly donated by Sankyo Co., Ltd. (Tokyo, Japan). Their structures and that of BF are shown in Fig. 1. All reagents were of special reagent grade.

Solubilities of HCRIs in Various Solvents Purified water, acetonitrile, methanol, ethanol, acetone, 2-propanol, ether, and chloroform were employed as solvents. An excess amount of HCRI $(1-100 \mathrm{mg} / \mathrm{dl})$ was put into a $30-\mathrm{ml}$ beaker, water or solvents were added, and the mixture was well stirred at $80 \mathrm{rpm}, 25^{\circ} \mathrm{C}$ for $2 \mathrm{~h}$. A previous pilot study had confirmed that equilibrium was reached within $2 \mathrm{~h}$. The suspension was filtered with a Millipore filter (Type PVDF; $0.45 \mu \mathrm{m}$, Tokyo, Japan) and the filtered solution was diluted with solvent (mobile phase). The drug concentration of the diluted sample was determined using an HPLC method: $50 \mu \mathrm{l}$ was injected onto a chromatograph (Shimadzu SPD6A) equipped with an integrator (Shimadzu C-R4A) and a reversed phase column (Shim-pack CLC-CN, 6.0 $\times 150 \mathrm{~mm}$, Shimadzu Co., Ltd., for PV, NavaPak C18, 3.9 $\times 150 \mathrm{~mm}$, Waters Co., Ltd., for $\mathrm{SV}, \mathrm{CeV}, \mathrm{AV}$, and FV). The mobile phase employed was acetonitrile: water: triethylamine: glacial acetic acid (250:750:1:1 for PV, 500:500:1:1 for SV, $\mathrm{CeV}, \mathrm{AV}$, and $\mathrm{FV}$ ) and the flow rate was $1.2 \mathrm{ml} / \mathrm{min}$. The wavelength of $238 \mathrm{~nm}$ was selected. Column temperature was maintained at room temperature. 


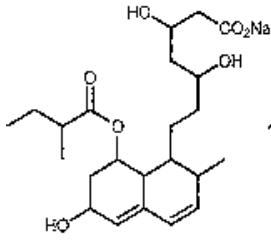

Pravastatin (PV)

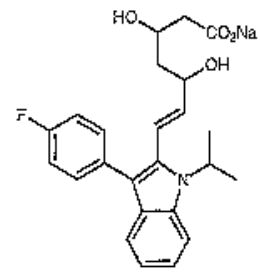

Fluvastatin $(F \mathrm{~V})$
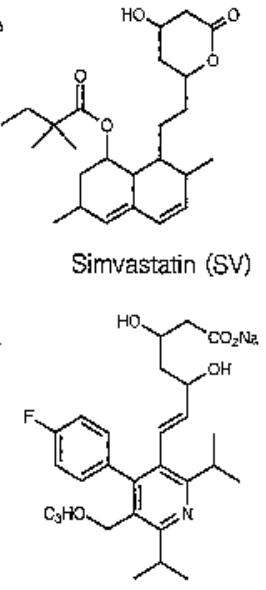

Cerlvastatin (Cew)

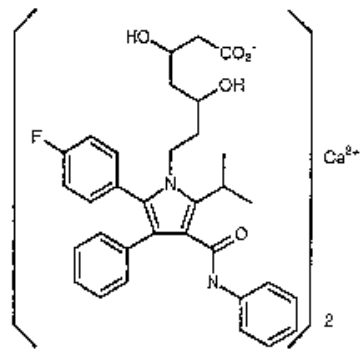

Adorvastatin (A)

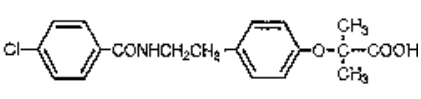

Bezafibrate $(B F$

Fig. 1. Chemical Structures of HMG-CoA Reductase Inhibitors and Bezafibrate

Table 1. Maximum Solubility Values (mg/ml) of Each HMG-CoA Reductase Inhibitor (HCRI) in Water, Acetonitrile, Methanol, Ethanol, Acetone, 2Propanol, Ether and Chloroform, together with Water/Octanol Partition Coefficients $(\log P)$

\begin{tabular}{|c|c|c|c|c|c|}
\hline \multirow{2}{*}{ Solvent } & \multicolumn{5}{|c|}{ Drug (Partition coefficient) } \\
\hline & $\mathrm{PV}(-0.47)$ & $\mathrm{AV}(1.53)$ & $\mathrm{FV}(1.75)$ & $\mathrm{CeV}(2.32)$ & SV (4.40) \\
\hline Water & $>100$ & 0.31 & 86.81 & $>100$ & $7.25 \times 10^{-5}$ \\
\hline Acetonitrile & $1.40 \times 10^{-5}$ & 0.29 & 0.19 & 0.17 & $>100$ \\
\hline Methanol & $>100$ & $>100$ & $>100$ & $>100$ & $>100$ \\
\hline Ethanol & 51.64 & 30.43 & $>100$ & $>100$ & $>100$ \\
\hline Acetone & $4.20 \times 10^{-2}$ & 79.14 & $>100$ & $>100$ & $>100$ \\
\hline 2-Propanol & 7.17 & $6.78 \times 10^{-2}$ & 0.88 & 93.78 & 79.55 \\
\hline Ether & $5.52 \times 10^{-4}$ & $3.62 \times 10^{-3}$ & $1.04 \times 10^{-2}$ & 0.34 & 19.09 \\
\hline Chloroform & $1.44 \times 10^{-3}$ & 75.49 & $>100$ & $>100$ & $>100$ \\
\hline
\end{tabular}

Determination of Plasma CPK Level in Normal Rats under Urethane Infusion Following Oral Administration of HCRIs or HCRIs + BF Male Wistar rats, aged 8 weeks old $(180-200 \mathrm{~g})$, were used. The rats were not allowed to drink liquid for a day. The day before the experiment, the jugular vein was cannulated. The rats were fasted overnight after the cannulation, then administered HCRI with or without BF. In the case of oral administration, all drugs were suspended in $0.5 \%$ aqueous methyl cellulose solution. HCRI was orally administered to rats via oral gavage at a dosing volume of $5 \mathrm{ml} / \mathrm{kg}$. Control animals were dosed in a similar manner with purified water. After dosing, the rats administered HCRI with or without BF were held in Boltsmann cages. One hour after oral drug administration, $25 \%$ urethane was intravenously infused to the rats via jugular cannulation at a constant rate of $5 \mu \mathrm{l} / \mathrm{min}$ for $3 \mathrm{~h}$ and then at $1 \mu \mathrm{l} / \mathrm{min}$. We had previously confirmed that in control rats, the plasma CPK level remains unchanged for $10 \mathrm{~h}$, after which CPK level gradually increases. Fifty microliters of blood was withdrawn from the jugular vein at $7 \mathrm{~h}$ after drug administration. The blood samples obtained were used to determine the CPK level, the skeletal muscle marker enzyme, by spectrophotometric measurement using a commercial kit (Dainabot Co., Ltd., Tokyo, Japan). After urethane infusion, the kidney was extracted from the rat, and renal tissue specimens were prepared as described in the following section.

Preparation of Renal Tissue Specimens and Observa- tion by Light Micrographs Rat kidney was soaked in 10\% formaldehyde solution, then embedded in paraffin. Thin paraffin sections $(2 \mu \mathrm{m})$ were deparaffinized in xylene and washed. To inhibit endogenous peroxidase, $3 \%$ hydrogen peroxidase was applied to the sample for $10 \mathrm{~min}$, followed by washing with TBST (Tris buffered saline with Tween 80) three times. Next, 10\% normal goat plasma was added, and the sample was left standing for 15 min. Rabbit anti human myogloblin polyclonal antibody was added to the sample, which was then left at $4{ }^{\circ} \mathrm{C}$ overnight. Biotinylated anti-rabbit immunoglobulin was applied to the section, which was then washed with TBST three times. Horseradish peroxidase-labelled streptoavidin was dropped onto the sample, with simultaneous washing with TBST. Next, $0.05 \%$ of $3,3^{\prime}$-diaminiobenzidine was dropped onto the section. Finally, Meyer's hematoxylin was added for nuclear staining. The thin section was rinsed gently with distilled water, then counterstained, dried, and mounted. The prepared sample was observed by optical microscopy (GHS type, Olympus Co., Ltd.,Tokyo, Japan).

\section{RESULTS}

Solubilities of HCRIs in Various Solvents Table 1 summarizes the solubilities in various solvents or partition coefficients for each drug. ${ }^{14)} \mathrm{PV}$ has a high solubility for water, but shows poor solubility for non-polar solvents such 
as acetonitrile, acetone, ether, and chloroform. On the other hand, $\mathrm{CeV}$ or $\mathrm{SV}$ with a large partition coefficient shows large solubilities for non-polar solvents such as 2-propanol, ether, or chloroform. These data seem to suggest that the solubility of each drug coincides well with its water/n-octanol partition coefficiency. From the standpoint of lipophilicity, the drugs could be ranked in the following order: SV $>$ $\mathrm{CeV}>\mathrm{FV}>\mathrm{AV}>\mathrm{PV}$.

Plasma CPK Level in Normal Rats under Urethane Infusion Following Oral Administration of HCRIs alone or HCRIs with BF Figure 2 shows the plasma CPK levels after oral administration of HCRI alone (A) or with $50 \mathrm{mg} / \mathrm{kg}$ of BF (B). On administration of HCRI alone, as shown in Fig. 2A, the CPK level increased as the HCRI dose increased. From the standpoint of the CPK level, the risk of myopathy was ranked in the following order: $\mathrm{CeV}>\mathrm{FV}>$ $\mathrm{AV}>\mathrm{SV}>\mathrm{PV}$. In the case of PV, the CPK level was not enhanced as the dose increased and remained very low. However, as shown in Fig. 2B, coadministration of BF with HCRI increased the CPK level 1.5- or 2-fold compared with the administration of HCRI alone.

Histopathological Findings of Kidney Treated with HCRIs under Urethane Infusion Myoglobin deposition was also observed in the cast of proximal tubules, cytoplasm of distal tubules and collecting ducts of kidney from rats administered HCRIs or coadministered BF even though the extent of myoglobin deposition differed. Figure 3 shows the light micrographs of kidney extracted from male rats treated with saline (Fig. 3A) as a negative control, $50 \mathrm{mg} / \mathrm{kg}$ BF (Fig. 3B), $100 \mathrm{mg} / \mathrm{kg} \mathrm{PV} \mathrm{(Fig.} \mathrm{3C),} 100 \mathrm{mg} / \mathrm{kg} \mathrm{AV} \mathrm{(Fig.} \mathrm{3D),} 100$ $\mathrm{mg} / \mathrm{kg} \mathrm{FV}$ (Fig. 3E), $100 \mathrm{mg} / \mathrm{kg} \mathrm{CeV} \mathrm{(Fig.} \mathrm{3F),} 100 \mathrm{mg} / \mathrm{kg}$ SV (Fig. 3G) and $100 \mathrm{mg} / \mathrm{kg} \mathrm{FV}$ plus $50 \mathrm{mg} / \mathrm{kg} \mathrm{BF} \mathrm{(Fig.} \mathrm{3H)}$ under urethane infusion respectively. Any myoglobin disposition was not observed in rats treated with saline as shown in Fig. 3A. In the case of BF (Fig. 3B) as a positive control, moderate myoglobin disposition was observed in the cast of proximal tubules, and the cytoplasm of proximal or distal tubules, and desquamation was found for the proximal tubular cells. Among the HCRIs, PV causes the least change in myoglobin disposition in the cast of proximal tubules and in the cytoplasm of proximal or distal tubules as shown in Fig. $3 \mathrm{C}$. In the case of $\mathrm{SV}$, a comparatively slight change was observed (Fig. 3G). In the case of AV (Fig. 3D), FV (Fig. 3E), or $\mathrm{CeV}$ (Fig. 3F), moderate or severe myoglobin deposition was observed in the rat kidney. As shown in Fig. 3D, marked myoglobin deposition observed in the cast of proximal tubules, moderate cytoplasm of proximal or distal tubules, and desquamation of proximal tubular cells were also observed in the kidney specimen treated with $100 \mathrm{mg} / \mathrm{kg}$ of FV. The extent of change was enhanced by coadministration of $\mathrm{BF}$ as shown in Fig. 3H. Thus relationship between CPK and histopathological finding was observed to some extent even though quantitative correlation was not obtained.

\section{DISCUSSION}

The disposition of drugs in humans depends on their physicochemical properties. The drug with high lipophilicity tend to cause myopathy since such drugs are easily partitioned to organs such as muscle. Therefore, the risk of myopathy seems to be proportional to the lipophilicity of
(A)

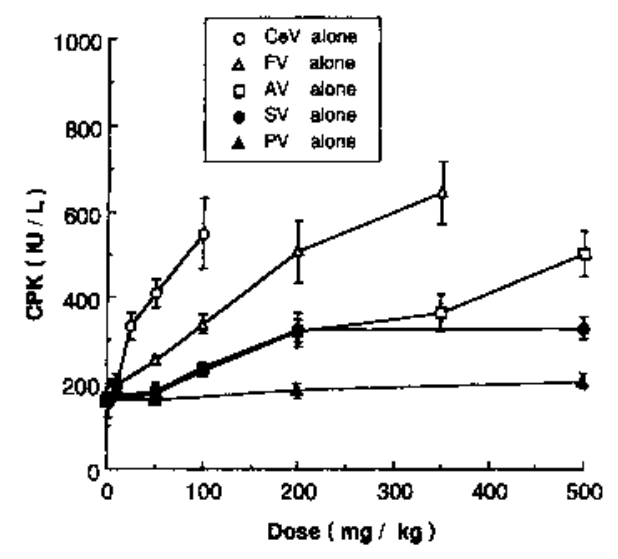

(B)

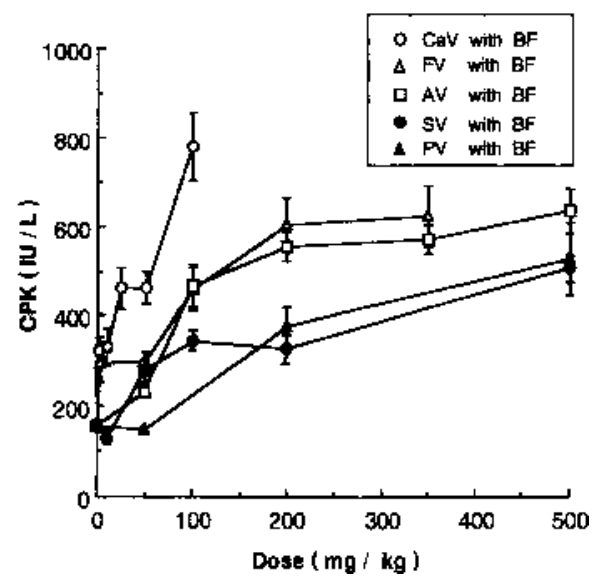

Fig. 2. Plasma CPK Levels after Oral Administration of HMG-CoA Reductase Inhibitors

Alone (A) or with (B) $50 \mathrm{mg} / \mathrm{kg}$ of BF. Each point represents the mean \pm S.E. of $4-9$ rats.

HCRIs. The solubility data which was adopted as a physicochemical property, as shown in Table 1, almost coincides with the water $/ n$-octanol partition coefficient. The lipophilicities of HCRIs was ranked in the following order: $\mathrm{SV}>\mathrm{CeV}>$ $\mathrm{FV}>\mathrm{AV}>\mathrm{PV}$.

By urethane infusion method, as shown in Fig. 2A, the risk of myopathy evaluated by the CPK level was ranked in the following order: $\mathrm{CeV}>\mathrm{FV}>\mathrm{AV}>\mathrm{SV}>\mathrm{PV}$. The correlation was found for myopathy risk evaluated by CPK level obtained in the urethane infusion method and drug lipophilicity, i.e., the water $/ n$-octanol partition coefficient except for the case of SV. SV, which has high lipophilicity, did not elevate the CPK level as much as $\mathrm{CeV}$ or FV in the present study. One reason for this phenomenon seems to be the faster metabolic rate of SV. As shown in Fig. 4, SV was easily transformed to SVA, an open acid form, which is more hydrophilic than the parent compound. Therefore, the extent of partition of SVA does not seem to be very high. Among HCRIs, CeV most elevated CPK level in urethane infusion method as shown in Fig. 2. Even in a small dose of $\mathrm{CeV}$ could lead to myopathy. With FV and AV, the risk of myopathy increased as the dose increased. On the other hand, even though $\mathrm{FV}$ and $\mathrm{CeV}$ with high lipophilicity display a high risk of elevating the CPK level, these drugs have the advan- 

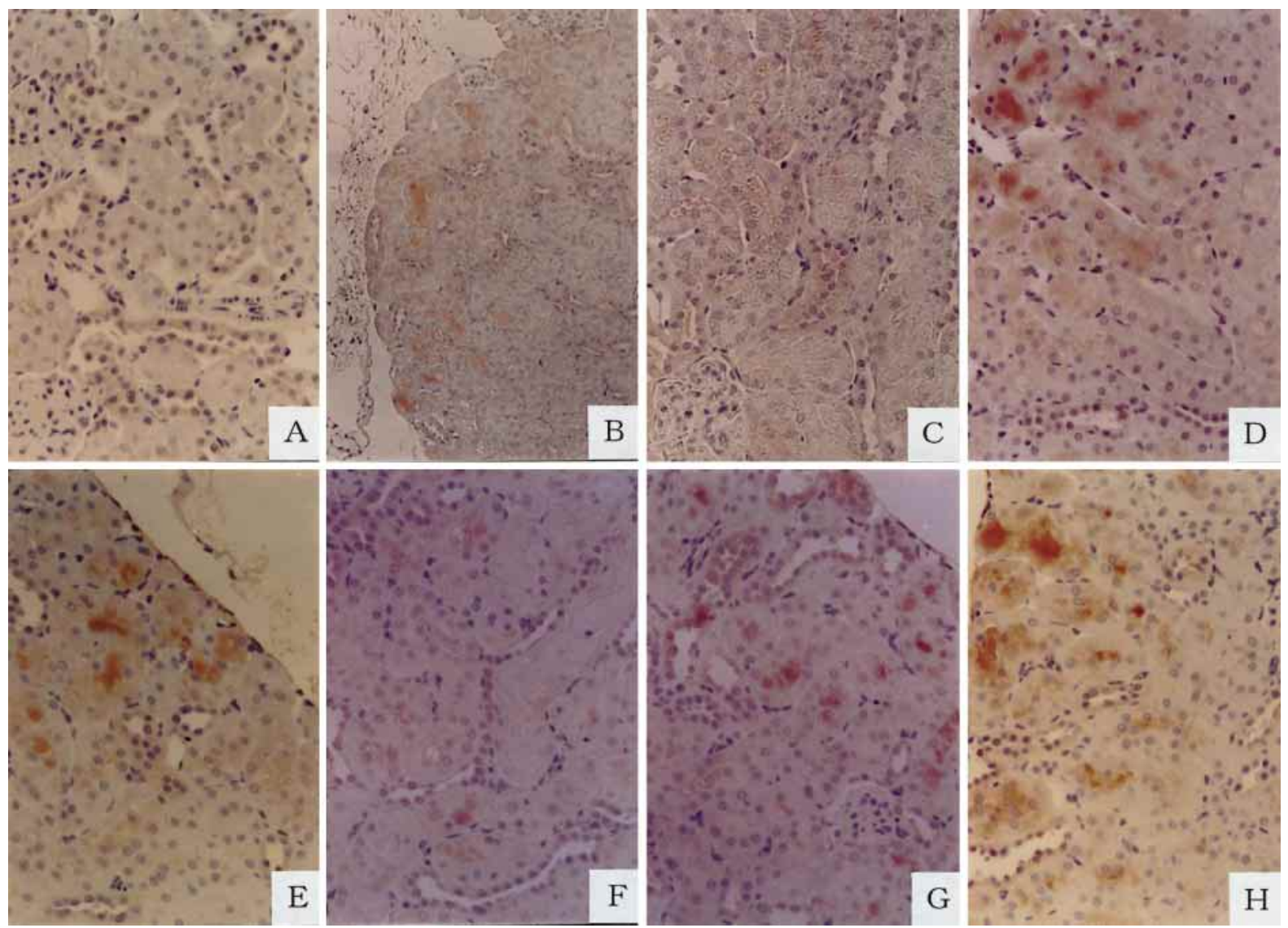

Fig. 3. Light Micrographs of Kidney Extracted from Treated Male Rats

Rats were given control (A), $50 \mathrm{mg} / \mathrm{kg} \mathrm{BF} \mathrm{(B),} 100 \mathrm{mg} / \mathrm{kg} \mathrm{PV}$ (C), and $100 \mathrm{mg} / \mathrm{kg} \mathrm{AV} \mathrm{(D),} 100 \mathrm{mg} / \mathrm{kg} \mathrm{FV} \mathrm{(E),} 100 \mathrm{mg} / \mathrm{kg} \mathrm{CeV} \mathrm{(F),} 100 \mathrm{mg} / \mathrm{kg} \mathrm{SV}$ (G), and 100 mg/kg FV plus $50 \mathrm{mg} / \mathrm{kg} \mathrm{BF}(\mathrm{H})$ by urethane infusion.

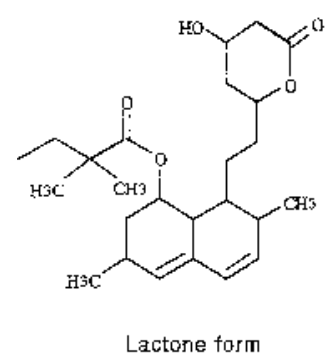

(SV)

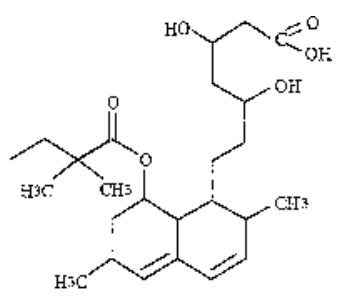

Hydroxy acid form

(SVA)

Fig. 4. Metabolic Tranformation from SV (Lactone Form) to SVA (Hydroxy Acid Form)

tage of accelerating the lipid metabolism in cardiac cells. ${ }^{15)}$

Whereas among the HCRIs, PV gives the lowest CPK level as shown in Fig. 2. One reason for this may be the high selectivity of PV to the level in drug disposition as described in the previous report, ${ }^{16)}$ with PV not inhibiting the synthesis of cholesterol in organs other than the liver. In addition, as previous reported, ${ }^{17)}$ HCRIs differs in metabolism pathway. For examples, lovastatin, SV, and AV are substrates of CYP3A4, whereas FV is metabolized by CYP2C9. Pravastatin is not extentively metabolized by either of these isoenzymes; rather, it is transported into hepatocytes by a sodiumindependent, carrier-mediated uptake system that normally transports bile acids. Compared with other HCRIs, PV thus has a reduced potential for drug interactions with other substrates. However, in a patient undergoing artificial dialysis,

severe myopathy and rhabdomyolysis have been reported after a single oral administration of $\mathrm{PV},{ }^{18)}$ and attention must be paid to the risk of myopathy.

Concomitant use of fibrates with HCRIs has been associated with a increased risk of myopathy, but the underlying mechanism of myopathy remains unclear. In the present study, as shown in Figs. 2A and B, in the case of $\mathrm{CeV}$ and $\mathrm{FV}$, the plasma CPK level was further enhanced by coadministration of $\mathrm{BF}$ even at low doses. In the case of single oral administration of PV, the CPK level was not enhanced, but coadministration of $\mathrm{BF}$ elevated $\mathrm{CPK}$ to the same level as $\mathrm{SV}$. In the case of SV, coadministration of BF also elevated the CPK level as shown in Fig. 2. The synergic effect of BF in myopathy risk seems to be caused by a pharmacodynamic or pharmacokinetic drug-drug interaction in vivo. In general, the pharmacokinetics of HCRIs are very complicated. ${ }^{19,20)}$ For example, the elimination or metabolic rate has been reported to differ between young and elderly patients. ${ }^{21)}$ The another study reported that plasma concentrations of active lovastatin acid are markedly increased by gnfirozil by not by $\mathrm{BF}$ in human study. ${ }^{22}$ Even though we did not examine the effect of concomitant treatment of HCRIs and fibrates on plasma concentration level of HCRIs, the enhancement of CPK by concomitant treatment with BF in the present study may be partially of a pharmacokinetic origin. We are going to examine the mechanism of synergic effect of myopathy risk of concomitant use of fibrates with HCRIs on the basis of pharmacokinetic and pharmacological study. Especially 
we will try the interactive study using the cultured muscle cell.

In related to histopathological findings of kidney treated with HCRIs or BF almost coincided with CPK rank. Especially, the extent of myoglobin deposition evaluated visually almost reflect on the myopathy risk. In general, the myopathy risks of HCRIs were reported to be lower than that of BF, and coadministration of BF enhanced the risk of myopathy. ${ }^{3)}$ In fact, in the present study, the myoglobin deposition in renal tissue as a histopathological findings well proportional to the CPK level induced our urethane infusion model. For example, in the case of FV, coadministration of BF enhanced myopathy risk not only in the CPK level in low doses (Fig. 2), but also in the extent of myoglobin deposition (Figs. 3E, H).

Therefore, histopathological findings of the kidney obtained using this urethane infusion method almost correlated the extent of CPK level to some extent, and our urethane infusion method is expected to be convenient and useful method for evaluating the risk of myopathy of a variety of drugs such as other HCRIs, fibrates and other compounds.

Recently $\mathrm{CeV}$ products were withdrawn from Japanese drug market. The development and validity of the screening method for myopathy of various drugs is important.

Acknowledgements We thank Ms. Mitsue Morikawa for her technical assistance.

\section{REFERENCES}

1) Shepherd J., Cobbe S. M., Ford I., Isles C. G., Lorimer A. R., MacFarlane P. W., McKillop J. H., Packard C. J., N. Engl. J. Med., 333, 1301-1307 (1995).

2) The Scandinavian Simvastatin Survival Study Group, Lancet, 344,
1383 -1389 (1994).

3) Nakai A., Nishikata M., Uchida T., Ichikawa M., Matsuyama K., Biol. Pharm. Bull., 20, 104-106 (1997).

4) Lopez J. R., Alamo L., Capto C., Wikinski J., Ledezma D., Muscle \& Nerve, 9, 85-88 (1986).

5) Nakai A., "Drug-Drug Interaction among Hyperlipidemia Medicines," The doctoral dissertation of Mukogawa Women's University, 1997, p. 45.

6) Kalow W., Britt B. A., Terreau M. E., Haist C., Lancet, 2, 895-898 (1970).

7) Tsujita Y., Kuroda M., Shimada Y., Tanzawa K., Arai M., Kaneko I., Tanaka M., Masuda H., Tarumi C., Watanabe Y., Biochem. Biophys. Acta, 877, 50-60 (1986).

8) Vickers S., Duncan C. A., Chen I. W., Rosegay A., Duggan D. E., Drug Metab. Dispos., 18, 138-145 (1990).

9) Vickers S., Duncan C. A., Vyas K. P., Kari P. H., Arison B., Prakash S. R., Ramjit H. G., Pitzenberger S. M., Stokker G., Duggan D. E., Drug Metab. Dispos., 18, 476-483 (1990).

10) McClellan K. J., Wiseman L. R., McTavish D., Drugs, 55, 415-420 (1998).

11) Bocan T. M. A., Ferguson E., McNally W., Uhlendorf P. D., Bak Mueller S., Dehart P., Sliskovic D. R., Roth B. D., Krause B. R., Newton R. S. Biochem. Biophys. Acta, 1123, 133-144 (1992).

12) Posvar E. L., Radulovic L. L., Cilla D. D., Jr., Whitfield L. R., Sedman A. J., J. Clin. Pharmacol., 36, 728-731 (1996).

13) Hoffman W. F., Alberts A. W., Anderson P. S., Chen J. S., Smith R. L., Willard A. K., J. Med. Chem., 29, 849-852 (1986).

14) Shimada Y., Progress in Medicine, 18, 957-962 (1998).

15) Koga T., Shimada Y., Kuroda M., Tsujita Y., Hasegawa K., Yamazaki M., Biochem. Biophys. Acta, 1045, 115-120 (1990).

16) Hatanaka T., Clin. Pharmacokinet., 39, 397-412 (2000).

17) Beaird S. L., J. Am. Pharm. Assoc., 40, 637-644 (2000).

18) Ministry of Health and Welfare of Japan, "Information on Drug Adverse Reaction," No. 119, Tokyo, 1993.

19) McTavish D., Sorkin E. M., Drugs, 42, 65-89 (1991).

20) Todd P. A., Goa K. L., Drugs, 40, 583-607 (1990).

21) Lea A. P., McTavish D., Drugs, 53, 828-847 (1997).

22) Kyrklund C, Backman J. T., Kivisto K. T., Neuvonen M., Laitila J., Neuvonen P. J., Clin. Pharmacol. Ther., 69, 340-345 (2001). 